\title{
A Simplified Technique for Retrofitting a Post and Core Foundation to a Pre-existing Crown: A Case Report
}

\section{Saafi J*, Elamri S, Alqahtani J, Assiri MA and Al-ojaym T \\ Faculty of Dentistry, University of Monastir, Tunisia}

*Corresponding author: Saafi Jilani, Faculty of Dentistry, University of Monastir, Avicenna Street Monastir, Tunisia, Tel: +21623407311/ +21650407311/ +966549694345; Email: saafi_jilani@yahoo.fr

\section{Case Report \\ Volume 3 Issue 2}

Received Date: April 05, 2018

Published Date: April 23, 2018

DOI: $10.23880 /$ oajds-16000171

\section{Abstract}

Esthetic and functionally restoration of endodontically treated tooth is commonly performed by a post and core. Many causes of failure can lead to fracture of abutment such as composite debonding, trauma and recurrent caries. This paper describes a simplified technique for fabrication of a casted post and core by retrofitting an old metal ceramic crown using duralay resin as model without the original die or its replica in a simple and time-saving way.

Keywords: Abutment; Fracture; Retrofitting; Duralayresin; Occlusion; Casted post and core

Abbreviations: MI: Maximum Intercuspation; PVS: Polyvinyl Siloxane.

\section{Introduction}

Fracture of an endodontically treated and restorated tooth by a crown in the esthetic zone present a challenge to clinician [1,2]. A patient will generally want a cosmetic and functional prosthesis at the earliest possible opportunity [3]. Rebuilding the core followed by making an impression for a new crown fabrication may be an option, but it is time-consuming and financially demanding [4]. Over the years, a variety of techniques have been developed and suggested by different authors to retrofit the crown [5-8]. Depending on the extent and severity of the fracture, retrofitting the crown on a fractured core may be a preferred option [4]. Nevertheless, the existing crown should fit precisely on the pre-existing finish line [9].

\section{Case Report}

A 40-year healthy female presented to Aseer specialist dental center in emergency with a loss of esthetic crown related to the maxillary premolar (Figure 1).

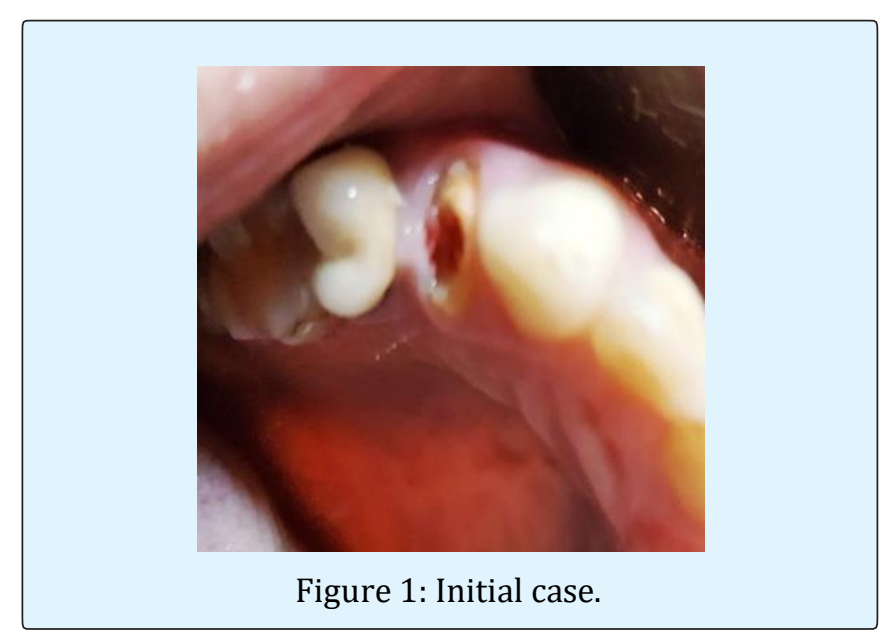


The patient sleeked for a quick and urgent aesthetic and functional solution. On examination, Patient had no medical history. Dental history did not reveal any past present symptom. A metal ceramic crown overlying the maxillary right first premolaras extracoronal full coverage prosthesis was seen dislodged since two weeks and remaining intact, whereas elsewhere a gingival hypertrophy has developed in the distal face of this tooth. The maxillary right first Premolar was decayed with sound dentine height of less than $1 \mathrm{~mm}$ (arrow) between the remaining already prepared tooth and crown margins (Figure 2). It was endodontically treated and with a remaining screw post in the post space of the buccal canal (Figure 3).

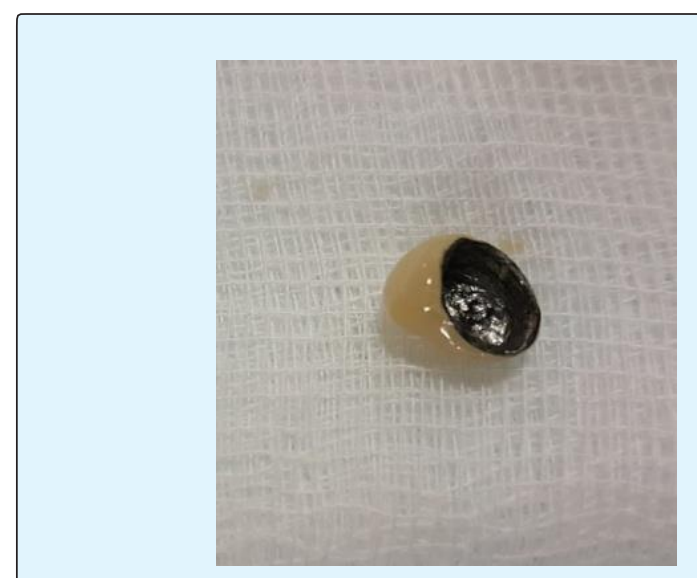

Figure 2: Pre-existence metal ceramic crown.

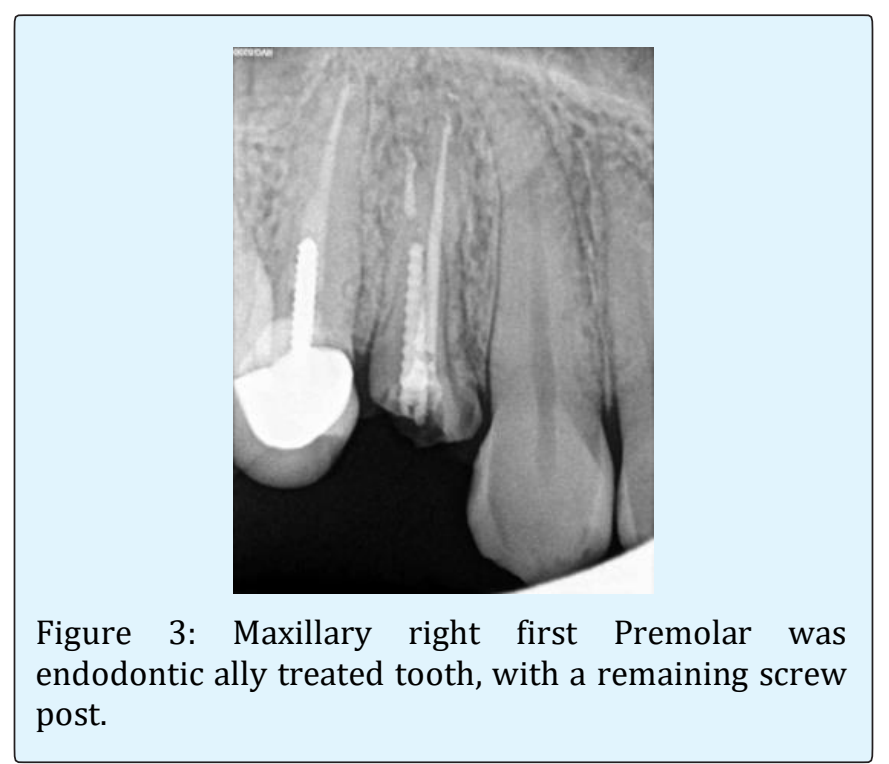

A Simplified Technique for Retrofitting a Post and Core Foundation to a Pre-existing Crown: A Case Report. J Dental Sci 2018, 3(2): 000171.
The patient was given the option of performing a new prosthesis or retrofitting (or retrograde) a casted post and core and recementing the same prosthesis. The patient choose to have the same prosthesis recemented, due to the costs involved in the fabrication of a new metal ceramic crow and duration of other treatment plan. Before reusing the existing intact prosthesis, a thorough investigation is mandatory to rule out any root fracture, residual caries, existing restoration and violation of the biologic width due to trauma or damage to the supporting tissues. First the screw post was removed by vibrating with an ultrasonic scaler. After the coronal portion of the post has been well isolated, the tip of the ultrasonic device is placed against it, and energy is applied to disrupt the cement interface which weakened brittle cement and facilitates removal. On another hand the remaining composite and dentine have been removed from the inner surface [10].

Moreover the patient has undergone a gingivectomy (Figure 4) to ensure a distal crown lengthening In order to exhibit the finish line that can be used to maintain periodontal health and long-term stability of the restorations.

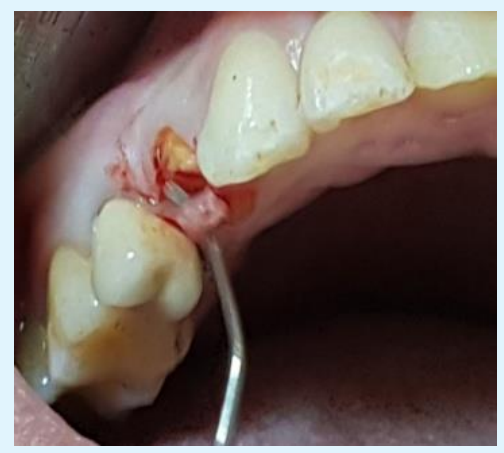

Figure 4: Surgical crown lengthening.

The interim restoration was performed by two techniques: by Using the existing prosthesis as a provisional restoration which was lined with autopolymerising resin (Figure 5) or a preformed polycarbonate crown which needed addition by the lining procedure and some modification (internal relief, axial recontouring, occlusal adjustment) (Figure 6) and each technique needed a metal post to improve retention which was inserted in the remaining post space already under taken for the old screw post. Thus the interim crown was a temporary post crown (Figure 7) which provides occlusal function, protection of the tooth structure ,ability to be cleaned, and restores function and 
esthetics during healing [11]. The provisional crown was cemented in place using non-eugenol temporary cement.

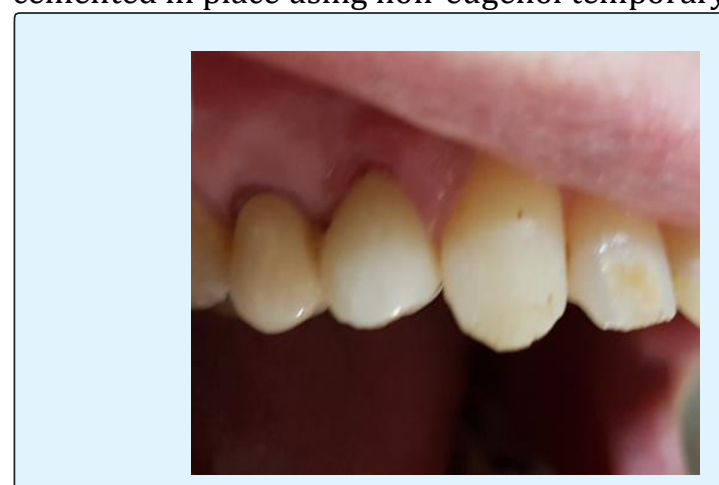

Figure 5: Using the existing prosthesis as a provisional.
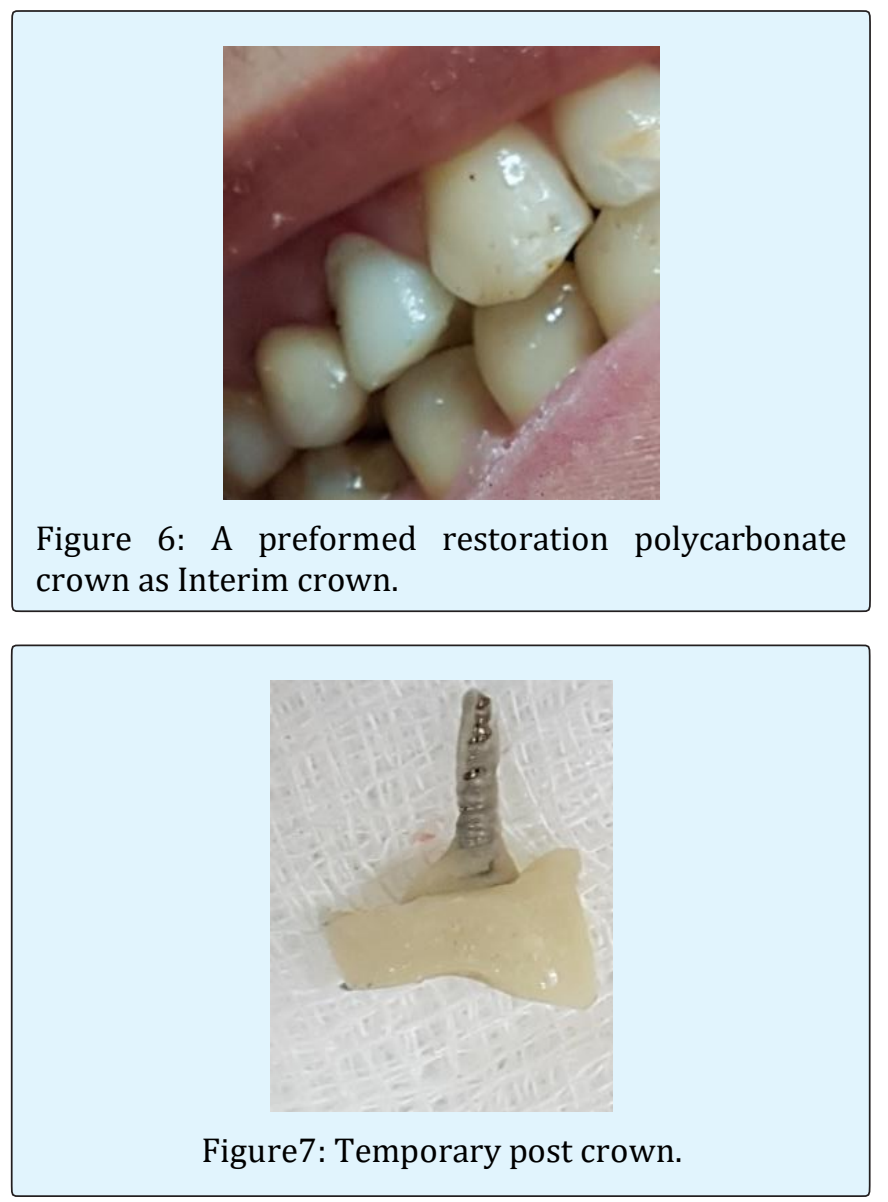

For post space preparation the enlargement of the canal was done using Peeso Reamers (sizes 1 to 3 ). Minimal enlargement and shaping of the canal is advised during post preparation due to anatomical considerations including thin mesio-distal dimensions and proximal root invaginations [12]. The residual cement in the post space was removed and the prepared space was cleaned with mouth wash.

The inner surface of the old metal ceramic crown was cleaned using a slow speed number 6 round bur (SS White Burs, Inc, USA), cutting through bulk of the fractured tooth fragment and the old remaining composite build up within the crown moving from center outward. A care must be taken to prevent the contact between the rotating bur and the inner surface of the crown [13].

After three weeks the healing of the gum related to the gingiva was well improved .Once selecting an appropriate size of plastic post (Para-Post, Whale dent International, New York, N.Y.), it was inserted into the prepared root canal. The length of the post was adjusted by cutting with a bur (Figure 8) so it did not interfere with the inner surface of the crown which may compromise accurate seating and appropriate occlusion.

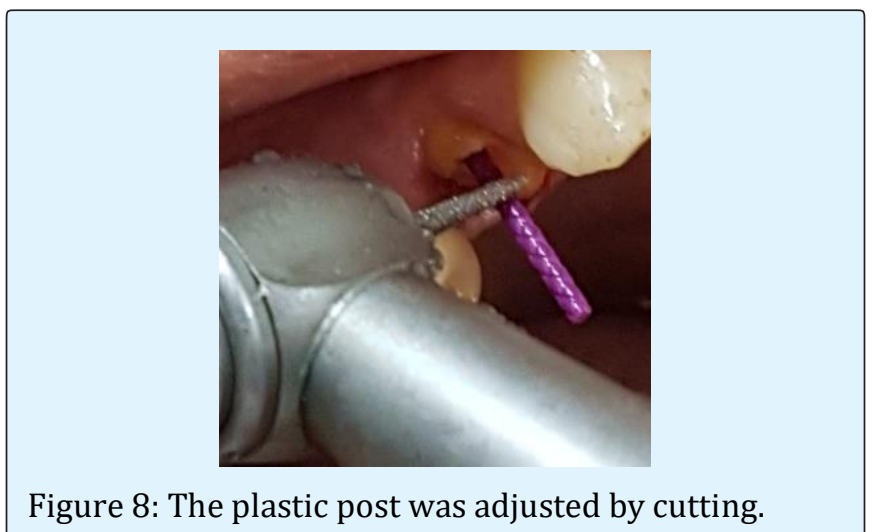

After applying a layer of lubricant to the tooth structure an autopolymerizing acrylic resin material (Duralay, Reliance Dental Mfg Co, Alsip, Ill.) was used to rebase the plastic post and to coatits coronal part to ensure better adaptation to the tooth flared canal and pulp chamber structure (Figure 9).

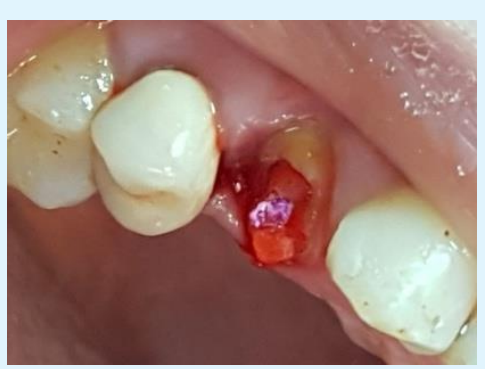

Figure 9: The plastic post was rebased with duralay resin. 
The inner surface of the crown was lubricated with petroleum jelly, which allowed for easy removal of the direct pattern made by resin duralay build up for the direct casted post and core. After mixing, the duralay resin material was filled into the inner aspect, then the patient was asked to close in the position of maximum intercuspation (MI) (Figure10) until the resin material completely polymerizes to avoid over-occlusion. Once the post and core pattern removed from the abutment tooth (sometimes from the existing crown), it was checked and any projection of material may remain over the margin was eliminated (Figures 10-12). It was also refined and sent to the lab for investing and casting (Figure13).

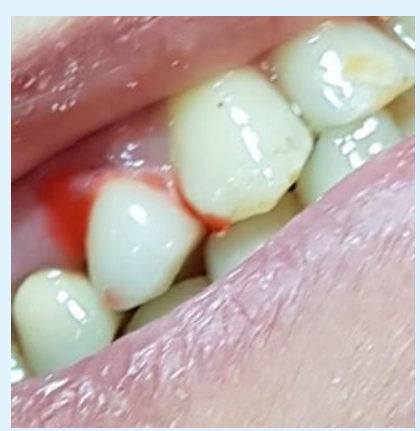

Figure 10: Post and core pattern performed.
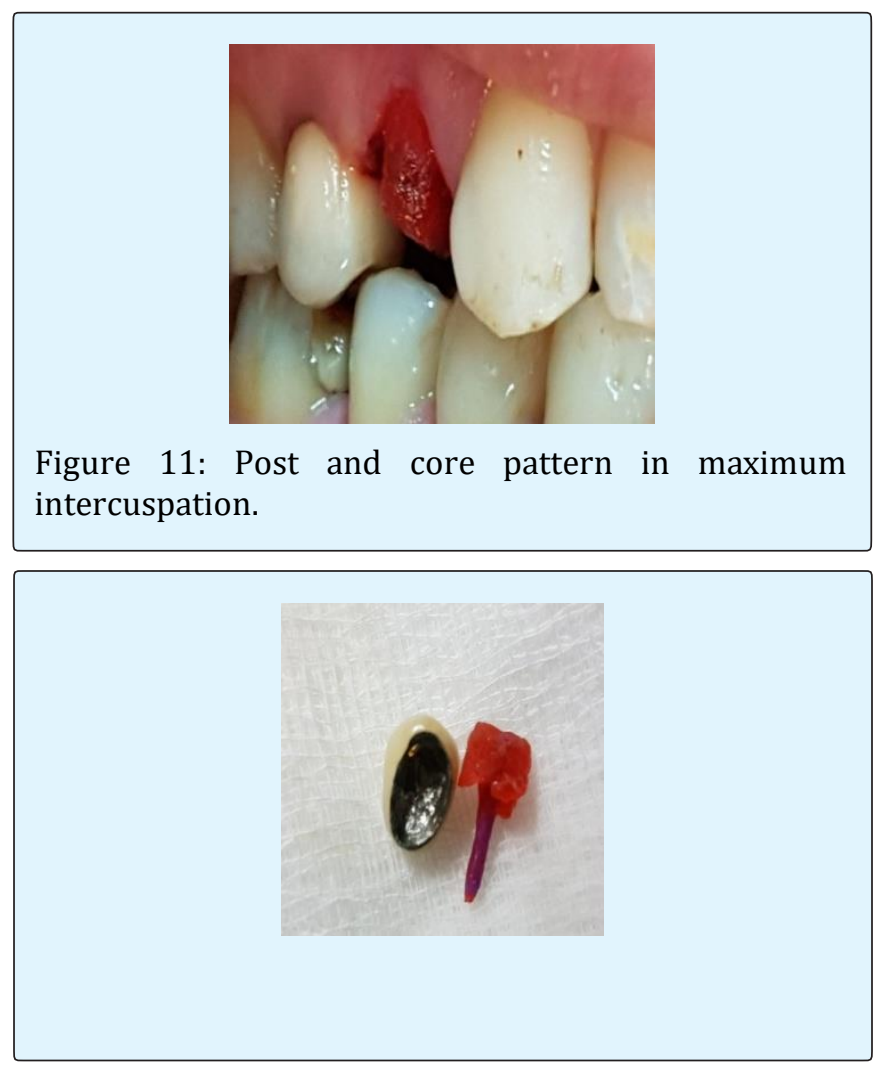

Figure 12: Post and core pattern removed from the abutment tooth.

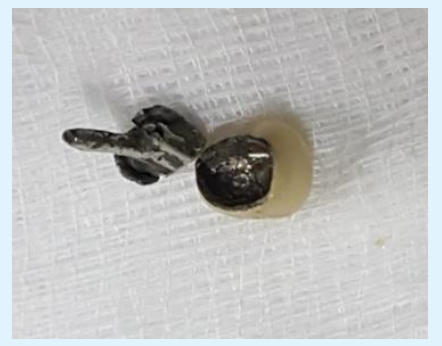

Figure 13: Existing crown and casted post and core after casting.

The casted post and cores was tried in the mouth without the crown (Figure 14) to ensure the perfect adaptation, then in a second time with the crown in situ to confirm fit, insertion, retention, marginal integrity, and to verify the occlusion during static and functional movements (Figure 15). Adequate cement thickness was ensured by the finishing procedures of the casted post and core that respected the existing tooth margins.

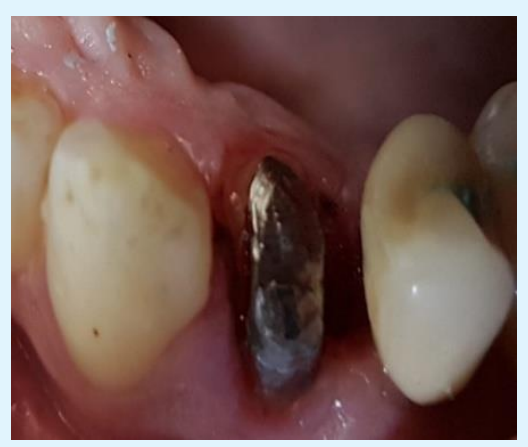

Figure 14: Try in casted post and core without.

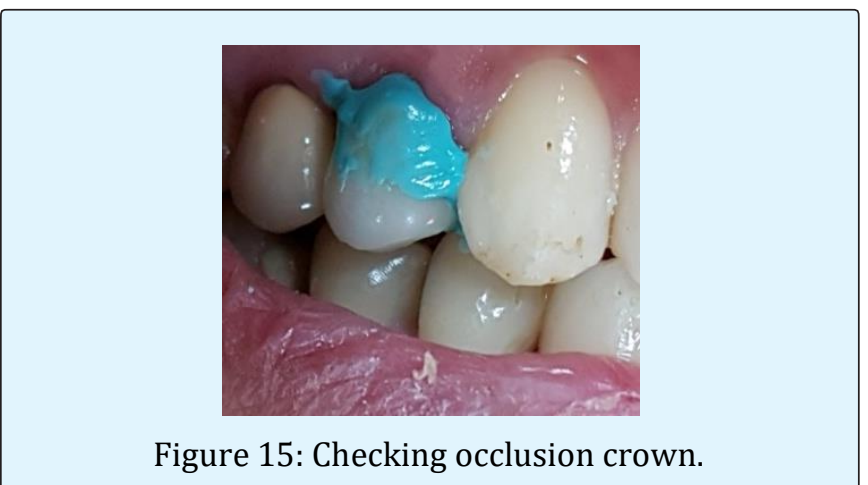

Figure 15: Checking occlusion crown. 


\section{Open Access Journal of Dental Sciences}

In the clinic the casted post and cores and the existing old metal ceramic crown were cemented simultaneously using adhesive cement (Figure 16).

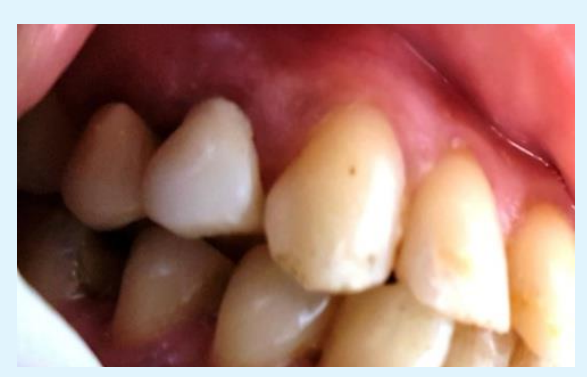

Figure 16: Cementation of the pre-existing crown.

\section{Discussion}

This article describes a procedure of retrofitting the existing crown to repair a fractured abutment using direct pattern made by resin duralay buildup [14]. Various authors have suggested different approaches to retrofit the crown [5-8]. Chan [6] adapted a polytetrafluoroethylene sheet as a separating medium onto the inner aspect of the crown. With this technique, partially set core material may get distorted and it also leaves additional cement space between the crown and the core. Jahangiri \& Feng [5] used the original die or its replica and a vinyl polysiloxane matrix preparing the autopolymerizing resin pattern to repeat casting of the postcore. Berksun [8] used manually fabricated plastic foil as a template for rebuilding the core, but there is a chance of distortion of the foil adapted manually. Sabbak [7] used a clear polyvinyl siloxane (PVS) impression material as a matrix. The intensity of light to cure the underlying composite resin may get reduced through bulky PVS. The existing crown should fit precisely on the pre-existing finish line. The repaired core should be placed exactly in the same location as the previous core. Slight change in core location may change the path of insertion of the crown Jeopardizing its adequate stability and retention [9]. The amount of remaining tooth structure appears to be of major importance, as the residual dentin helps in incorporating a ferrule. Only few studies have demonstrated that the presence of tooth structure coronal to the finish line did not enhance fracture resistance $[15,16]$.

A minimum sound dentine height of $1.5-2 \mathrm{~mm}$ was required between the core and crown margins. The final restoration provided a bracing, casing or hugging action to improve the integrity of the endodontically treated tooth [17].
Both amalgam and bonded composite cores required the presence of a minimum of $1.5-2 \mathrm{~mm}$ height of ferrule after crown preparation [18]. In our clinic case the abutment was very dilapidated. The remaining sound dentin (ferrule) in the distal surface was, as previously mentioned above, less than $1 \mathrm{~mm}$, so a casted post and core foundation was a suitable option. The advantages of using the existing prosthesis is, not only that the patient was psychologically satisfied by her old crown, but also the prosthesis incurred less occlusal wear versus an acrylic restoration and less time was required for fabricating a new temporary restoration.

In this case the procedure of bead- brush technique for recording contours of cast posts and cores using duralay resin was useless, because retrofitting the inner surface of a retainer should be under taken under occlusal pressure (maximum intercuspation) to procure a prosthesis with an occlusion entirely in harmony with the stomatognathic system. During the polymerization process, acrylic duralay resin materials has undergone volumetric contraction (shrinkage $95 \%$ before 3 hours at room temperature) [19]. It was due to of a high monomer/polymer ratio. In our technique Duralay resin was used in a thick consistency as was recommended by manufacturer and the excessive use of monomer was avoided as much as possible. Nevertheless it was not necessary to refit Duralay pattern set for several hours because, most of the shrinkage of Duralay resin $(80 \%)$ is expected before 17 minutes [7].

\section{Conclusion}

The technique elucidated resulted in a precise retrofitted casted post and core foundation which adapted well to the pre-existing crown. It used only minimum materials, affordable, time saving and was preferred by this patient over the other treatment options proposed. A three years follow up of this case has shown a successful outcome.

Source of Support: None, Conflict of Interest.

Acknowledgement: We would like to acknowledge all the team of the lab in Aseer specialist dental centre in Abha (KSA).

\section{References}

1. Rosen H (1998) Dissolution of cement, root caries, fracture, and retrofit of post and cores. J Prosthet Dent 80(4): 511-513. 


\section{Open Access Journal of Dental Sciences}

2. Lee JH (2017) A Digital Approach to Retrofitting a Post and Core Restoration to an Existing Crown. J Prosthodont.

3. Hannon SM, Breault LG, Kim AC (1998) The immediate provisional restoration: a review of clinical techniques. Quintessence Int 29(3): 163-169.

4. Patil PG, Tay K (2016) Modified technique to retrofit the crown on fractured core. J Interdiscip Dentistry 6(1): 50-53.

5. Jahangiri L, Feng J (2002) A simple technique for retrofitting a post and core to a crown. J Prosthet Dent 88(2): 234-235.

6. Chan DC (2003) Technique for repair of multiple abutment teeth under pre-existing crowns. J Prosthet Dent 89(1): 91-92.

7. Sabbak SA (2000) Simplified technique for refabrication of cast posts and cores. J Prosthet Dent 83(6): 686-687.

8. Berksun S (2005) Rebuilding core foundations for existing crowns using a custom-made template. J Prosthet Dent 93(2): 201-203.

9. Mascarenhas K, Aras MA, Fernandes AS (2013) Repair of fractured abutment teeth under pre-existing crowns: An alternative approach. Indian J Dent Res 24(1): 136-138.

10. Rosenstiel Land Fujimoto (2006) Contemporary fixed prosthodontics ISBN-13: 978-0323-02874-5 ISBN-10: 0-323-02874-8 tome 1 Copyright (C) 2006, 2001, 1995, 1988 by Mosby, Inc., an affiliate of Elsevier Inc pp: 371: 372.

11. Tseng SC, Fu JH, Wang H (2011) Immediate temporization crown lengthening. Compend Contin Educ Dent 32(3): 38-43.
12. Manning KE, Yu DC, Yu HC, Kwan EW (1995) Factors to consider for predictable post and core build-ups of endodontically treated teeth. Part 1: Basic theoretical concepts. J Can Dent Assoc J 61(8): 685-695.

13. Bhandari S, Rajagopal P, Bakshi S (2011) An interdisciplinary approach to reconstruct a fractured tooth under an intact all ceramic crown: Case report with four years follow up. Indian J Dent Res 22(4): 587-590.

14. Signore A, Benedicenti S, Kaitsas V, Barone M (2010) Simplified technique for rebuilding a post and core foundation with a preexisting crown: A case report. Quintessence Int 41(3): 205-207.

15. Al-Hazaimeh N, Gutteridge DL (2001) An in vitro study into the effect of the ferrule preparation on the fracture resistance of crowned teeth incorporating prefabricated post and composite core restorations. Int Endod J 34(1): 40-46.

16. Gegauff AG (2000) Effect of crown lengthening and ferrule placement on static load failure of cemented cast post-cores and crowns. J Prosthet Dent 84(2): 169-179.

17. Fernandes AS, Dessai GS (2001) Factors affecting the fracture resistance of post-core reconstructed teeth: A review. Int J Prosthod 14(4): 355-363.

18. Ng CCH (2006) Influence of remaining coronal tooth structure location on the fracture resistance of restored endodontically treated anterior teeth. J Prosthet Dent 95(4): 290-296.

19. Mojon P, Oberholzer JP, Meyer JM, Belser UC (1990) Polymerization shrinkage of index and pattern acrylic resins. J Prosthet Dent 64(6): 684-688. 